\title{
International Association of Trauma and Surgical Intensive Care Symposium: Penetrating Trauma. Update on Penetrating Trauma
}

\author{
Walter L. Biffl ${ }^{1}$
}

Published online: 4 April 2015

(C) Société Internationale de Chirurgie 2015

Significant advances in the management of penetrating trauma have taken place over the past decade. Military experience has demonstrated the benefits of changes in our prehospital management and resuscitation strategies, and improving technology has allowed an evolution in diagnostic and therapeutic paradigms. In the end, however, there are fundamental principles that continue to guide how we approach the patient with penetrating injury.

The trauma victim who suffers cardiopulmonary arrest is likely to die without immediate intervention. Dr. Civil and colleagues provide an overview of resuscitative thoracotomy in penetrating trauma, including indications, technical considerations, and outcomes. In addition, they discuss issues such as training, personnel, and locationpointing out that the capabilities in urban trauma centers in the US are not necessarily duplicated in hospitals worldwide. This is certainly a consideration in designing an institutional protocol for managing patients in extremis.

Penetrating neurotrauma can be immediately lethal or leave the victim with devastating disability. Rosenfeld and colleagues provide an overview of penetrating and blast injury to the central nervous system. Blast injury and its sequelae are being seen with increasing frequency in the aftermath of military conflict. The authors emphasize the importance of aggressive resuscitation and correction of coagulopathy, and highlight the need for early operative intervention in order to optimize outcomes. On the other

Walter L. Biffl

walter.biffl@dhha.org

1 Department of Surgery, Denver Health Medical Center/ University of Colorado, 777 Bannock St., MC 0206, Denver, CO 80204, USA hand, they offer criteria for nonoperative management of penetrating injuries to the brain and spinal cord. Finally, the importance of early decompression for blast injuries is highlighted.

In his paper on penetrating cervical trauma, Dr. Feliciano reviews the signs and symptoms of significant vascular or aerodigestive injury, and emphasizes that early operative intervention is most appropriate in symptomatic patients. Among patients without symptoms, he points out that serial clinical evaluation is accurate and that CT scanning does not improve on the accuracy. On the other hand, as he points out, CT scanning is likely to obviate the need for invasive testing in the patient with "soft" signs or equivocal exam findings. Finally, he offers valuable technical tips for the surgical management of cervical injuries.

In "Laparoscopy in penetrating abdominal trauma," Uranues and colleagues describe the benefits of employing technological advances in the diagnosis and management of abdominal trauma. As surgeons gain experience with laparoscopy, it can be relied upon to facilitate diagnosis of significant abdominal and thoracoabdominal injuriesparticularly diaphragmatic lacerations, small intestinal perforation, and mesenteric hemorrhage. The reduced surgical morbidity and length of stay, and cost-effectiveness of this approach compared with laparotomy, make a compelling argument for laparoscopy as the preferred approach in the stable patient. This paper highlights the importance of trauma surgeons' acquisition and maintenance of skills in the elective surgical realm, so that they may be applied in the emergency situation.

A different, even less-invasive, approach to penetrating abdominal trauma is described by Biffl and Leppaniemi. The authors offer evidence that, in patients with anterior abdominal stab wounds, performing additional testing 
often leads to nontherapeutic laparotomy and increased costs, without the added benefit of reduced morbidity related to (theoretically) earlier intervention. An algorithm for the management of stable patients with anterior abdominal stab wounds is presented. In addition, the conundrum of potential thoracoabdominal injuries is discussed. In order to identify all injuries and guide interventions, an algorithm based on physical exam, chest x-ray, and focused abdominal sonographic examination for trauma (FAST) is proposed.

Ivatury and colleagues have written a review on penetrating extremity trauma. They review established principles as well as newer developments in managing extremity vascular injuries. They emphasize the use of tourniquets when direct pressure does not control hemorrhage; immediate surgery when hard signs of vascular injury exist; and arterial imaging when there are no hard signs but the ankle:ankle or ankle:brachial index is abnormal. A number of technical details are offered, including the use of shunts and issues such as venous injuries, compartment syndrome, missile emboli, and the mangled extremity.

This symposium of articles should be read by all who manage trauma patients, as the papers are replete with useful, practical information 